\title{
Coupled river/estuary modeling approach for carbon dynamics in a temperate European river
}

\author{
GOULVEN G. LARUELLE ${ }^{1}$ AUDREY MARESCAUX ${ }^{2}$ \\ VINCENT THIEU ${ }^{3}$ JOSETTE GARNIER $^{4}$ \\ ${ }^{1}$ Université Libre de Bruxelles \\ goulven.gildas.laruelle@ulb.ac.be \\ ${ }^{2}$ Sorbonne Universités audrey.marescaux@upmc.fr \\ ${ }^{3}$ Sorbonne Universités vincent.thieu@upmc.fr \\ ${ }^{4}$ Sorbonne Universités josette.garnier@upmc.fr
}

At the interface between terrestrial and oceanic realms, rivers and estuaries collect, carry and transform large amounts of carbon and nutrients every year. Yet, the complex nature of these environments requires specific modeling strategies to resolve both the complex hydrological and biogeochemical dynamics that constrain these fluxes. Here, we present simulations performed using two Reactive Transport Models coupled to one another to reproduce in time and space the intricate dynamics of carbon, oxygen and nutrients along the main axis of a temperate european river: the Seine. The estuarine section is simulated by the generic estuarine model C-GEM (for Carbon Generic Estuarine Model), while the upstream part of the network, devoid of tidal influence, is simulated by the pyNuts-Riverstrahler modeling platform.

The Seine River discharges over $700 \mathrm{Gg}$ of carbon into the coastal sea every year, mostly under the form of dissolved inorganic carbon and emits $445 \mathrm{Gg}$ under the form of carbon dioxide $(\mathrm{CO} 2)$ into the atmosphere over its entire river network. This watershed, home to 18 million inhabitants, includes Europe's largest wastewater treatment plant and is submitted to large anthropic pressure. Our simulations provide a process-based spatio-temporal understanding of nutrients, oxygen, organic and inorganic carbon dynamics for the year 2010. Our coupled modeling chain allows quantifying the respective contributions of the estuarine and freshwater sections of the system in the removal of carbon as well as following the fate of TOC and DIC along the river network. Our results also allow calculating an integrated carbon budget of the Seine river network for the year 2010 